\title{
ARIAÇÃO SAZONAL DAS BORBOLETAS (LEPIDOPTERA) DA MATA do Museu de História Natural e Jardim Botânico da UfMG, Belo Horizonte, Minas Gerais, Brasil
}

\author{
Matheus Mir Leite Ferreira \\ Universidade Federal de Minas Gerais, Instituto de Ciências Biológicas, Avenida \\ Presidente Antônio Carlos, 6627, 31.270-901, Belo Horizonte, Minas Gerais, Brasil, \\ colgradc-bio@icb.ufmg.br
}

\section{André Roberto Melo Silva}

Centro Universitário UNA, Faculdade de Ciências Biológicas e Saúde, Rua Guajajaras, 175, Centro, 30.180-100, Belo Horizonte, Minas Gerais, Brasil

\section{Marina do Vale Beirão}

Universidade Federal de Ouro Preto, Instituto de Ciências Exatas e Biológicas, Rua Diogo de Vasconcelos, 122, 35.400-000, Ouro Preto, Minas Gerais, Brasil

\section{Gabryella Soares Pinheiro dos Santos}

Universidade Federal de Minas Gerais, Instituto de Ciências Biológicas, BirBrairLAB, Avenida Presidente Antônio Carlos, 6627, 31.270-901, Belo Horizonte, Minas Gerais, Brasil

\section{Mariana de Almeida Oliveira}

Universidade Federal de Minas Gerais, Instituto de Ciências Biológicas, Laboratório de Imunobiologia, Avenida Presidente Antônio Carlos, 6627, 31.270-901, Belo Horizonte, Minas Gerais, Brasil

\begin{abstract}
Resumo: As borboletas (Lepdoptera), por serem coloridas e de fácil visualização, são ótimas bandeiras para conservação. Também são boas indicadoras ambientais e úteis no monitoramento de ecossistemas. O objetivo desse estudo foi realizar análise da composição, riqueza e abundância das borboletas do Museu de História Natural e Jardim Botânico (MHNJB) da UFMG, relacionando esses parâmetros com a sazonalidade. As coletas foram realizadas bimestralmente durante um ano (entre junho de 2016 e maio de 2017). Cada amostragem foi realizada por dois dias, com o uso de redes entomológicas em cada uma das quatro áreas predeterminadas, num total de 48 horas de esforço amostral. Foram coletadas 571 borboletas de 95 espécies, sendo 40 singletons e 14 doubletons. A abundância e a riqueza não variaram entre os meses $(F=1,227, p=0,337 ; F=1,34, p=0,378$, respectivamente) e nem entre as estações $(F=0,7749, p=0,388 ; F=1,0045, p=0,327$, respectivamente). Já a composição das espécies diferiu entre os meses $(p=0,003)$ e entre as estações $(p=0,017)$. A curva de acúmulo de espécies não se estabilizou, indicando que há mais espécies para serem amostradas nas matas (áreas T1, T2, T3 e T4) do Museu.
\end{abstract}

Palavras-chave: ecologia, insetos, rophalocera, sazonalidade, urbanização.

SEAsonal Variation of the butterflies (Lepidoptera) from woods of the NatURAL History Museum and Botanical Garden of UfMG, Belo Horizonte, Minas Gerais, BRAZIL 


\begin{abstract}
Butterflies (Lepidoptera), because they are colorful and easy to see, are great flags for conservation. In addition, they are good environmental indications and useful in monitoring ecosystems. The objective of this study was to carry out an analysis of the composition, richness and abundance of butterflies from Natural History Museum and Botanical Garden (MHNJB) of UFMG, relating these parameters with a seasonality. The collections were carried out bimonthly for 12 months (between June 2016 and June 2017). Each sampling was carried out for two days using entomological nets in each of the four predetermined areas for a total of 48 hours of sampling. 571 butterflies of 95 species were collected, 40 singles and 14 doubles. Abundance and richness do not vary between months $(F=1,227, p=0,337 ; F=1,134, p=0,378$, respectively) or between seasons $(F=0,749, p$ $=0,88 ; F=1,045, p=0,327$, respectively). It already has a composition of different species between months $(p=0,003)$ and between seasons $(p=0,017)$. An unstabilized species accumulation curve, identified, as there are more species to be sampled in woods (T1, T2, T3 e T4) of the Museum.
\end{abstract}

Keywords: ecology, insects, rophalocera, seasonality, urbanization.

\section{INTRODUÇÃO}

$\mathrm{O}_{\mathrm{s}}$ lepidópteros, representados pelas borboletas e mariposas, incluem 160 mil espécies, o que corresponde a $16 \%$ dos insetos do mundo (Vanin, S.A, 2014). Suas características incluem um par de olhos compostos (Boggs et al., 2003), corpo, apêndices e asas cobertas por escamas pigmentadas ou cerdas, maxilas formando a espirotromba, como aparelho bucal sugador, metamorfose completa e larvas herbívoras (Beutel, 2014).

Dentro do grupo dos Lepidoptera, as borboletas estão divididas em duas superfamílias e seis famílias. A superfamília Papilionoidea inclui as famílias Papilionidae, Pieridae, Lycaenidae, Riodinidae e Nymphalidae, e a Hesperioidea, a família Hesperiidae (Ribeiro, 2010). Estes insetos são relativamente bem conhecidos, de fácil amostragem e possuem relações específicas com o habitat (Freitas et al., 2014).

As borboletas são segmentadas em duas guildas de acordo com a alimentação dos adultos. Algumas consomem néctar (nectarivoras) e outras se alimentam de frutas fermentadas e são consideradas frugivoras embora também haja ingestão de excrementos, exsudatos de vegetais e animais em decomposição (Filgueiras et al., 2016). Assim, os lepidópteros mantêm certa associação com determinadas espécies vegetais, realizando processos de polinização, herbivoria e decomposição. Essa polinização é, muitas vezes, essencial para o sucesso reprodutivo de uma planta (Verzoça \& Bion, 2011).

De modo geral, são excelentes indicadoras ambientais e muito úteis no monitoramento de ecossistemas já que demandam poucos recursos e, num curto prazo, para fornecimento de dados ecológicos (Oliveira et al., 2014). Esses fatores, aliados à alta sensibilidade às mudanças ambientais as tornam bons modelos para estudos sobre biologia da conservação, ecologia e evolução (Soga et al., 2015). Esse parâmetro de qualidade ambiental é especialmente eficaz em áreas fragmentadas ou que sofreram com intervenções humanas prolongadas. É o caso do Museu de História Natural e Jardim Botânico da UFMG (MHNJB-UFMG), que já foi fazenda, horto florestal, Instituto Agronômico e, atualmente, é uma área de preservação de Mata Atlântica secundária que apresenta diferentes estágios de renovação da vegetação.

Dos 33.100 ha que o município de Belo Horizonte ocupa, aproximadamente 4.000 ha (12\%) são compostos por áreas verdes $(\mathrm{PBH}$, 2009). Dentre elas, podem-se destacar também os Parques das Mangabeiras e Zoológico/Jardim Botânico. Tais locais são importantes para estabilização climática, já que diminuem oscilações térmicas (Breuste \& Artmann, 2015). Além disso, são eficientes na drenagem da água da chuva, servem de abrigo para a fauna sinantrópica e são excelentes espaços para lazer e educação ambiental (Chan et al., 2016).

A ecologia de comunidades busca entender quais são os mecanismos que geram padrões de riqueza e abundância das espécies na natureza. Para isso é preciso conhecer a estrutura das comunidades. Isso significa descobrir quantas espécies estão presentes, quais são esses organismos e em que número são encontrados para então relacionar com variáveis sazonais, temporais e biológicas, por exemplo (Ricklefs, 2003). Em áreas antropizadas e fragmentadas esse estudo é importante para inferir sobre a qualidade desses ambientes e propor medidas de conservação (Rocha et al; 2006). Entender quais os fatores influenciam na distribuição desses organismos é, portanto, essencial para o manejo mais eficiente dessa fauna confinada a pequenas porções de vegetação (Rocha et al., 2006). Além disso, ajuda a chamar atenção para que novos trabalhos de ecologia urbana sejam realizados nos parques da capital mineira com as borboletas e mariposas.

Assim, são os objetivos desta pesquisa conhecer a composição, a riqueza e a abundância da assembleia de borboletas da mata do 
MHBJB-UFMG e analisar a variação desses parâmetros através do tempo. Também tem como intuito complementar as informações previamente levantadas na área com as borboletas frugivoras - único estudo feito com esses insetos até o momento (Silva et al., 2014). Como hipótese, espera-se que nos meses mais frios e secos sejam coletados menos indivíduos e haja menor diversidade de borboletas quando comparado a época mais quente e chuvosa.

\section{Material e mÉtodos}

\section{Área de ESTUdo}

A pesquisa foi realizada na mata do $\mathrm{Mu}-$ seu de História Natural e Jardim Botânico da Universidade Federal de Minas Gerais (MHNJBUFMG), que se localiza em uma área de aproximadamente $600.000 \mathrm{~m}^{2}$ na região nordeste do município de Belo Horizonte (1953'29,8' S, $43^{\circ} 54^{\prime} 57,5^{\prime \prime}$ W) (Fig. 1) e é a terceira maior área verde da cidade (Felix, não publ.). Situada em uma região de Mata Atlântica, é composta por floresta estacional semidecidual. No museu há trilhas dentro das matas que são usadas para visitação e diversas atividades de educação ambiental. Nota-se na região a incidência de dois regimes climáticos, sendo os meses de outubro até março de maior precipitação e temperaturas elevadas e de maio a outubro o período mais frio e seco do ano (Climatempo). As temperaturas variam de $18{ }^{\circ} \mathrm{C}$ a $27{ }^{\circ} \mathrm{C}$ (Felix, 2009).

\section{Amostragem e identificação}

Realizaram-se coletas bimestrais no período de junho de 2016 e maio de 2017, num total de seis amostragens nesse período. Todas as etapas do projeto foram autorizadas pela diretoria do Museu assim como pelo Sistema de Autorização Informação em Biodiversidade SISBIO (n० 53728-1), com data de emissão em 25/05/2016 e de renovação em 24/06/2017.

As borboletas foram coletadas em quatro áreas distintas, com a utilização de duas redes entomológicas, considerando seu maior período de atividade (das $9 \mathrm{~h}$ às $17 \mathrm{~h}$ ). As áreas 1 e 2 são caracterizadas por serem de mata mais fechada. A área 3 possui região de borda da mata, mais aberta e ensolarada, e a área 4 compreende uma região de mata aberta e fechada. Cada área foi amostrada por 2 horas, o que resulta em 12 horas de esforço amostral em cada uma delas e 48 horas considerando as quatro áreas.

Cada amostragem foi realizada em um período de dois dias, divididos em manhã e tarde. Por exemplo, no primeiro dia, era feita a amostragem da área 1 de manhã e da área 2 à tarde e, no segundo dia, da área 3 de manhã e da área 4 à tarde. Além disso, os horários de coleta foram alternados entre as áreas, garantindo que todas as áreas de amostragem fossem percorridas nos dois períodos do dia ao longo do ano. As borboletas foram coletadas (Fig. 2A) e, quando possível, marcadas com caneta permanente e soltas. Dois exemplares de

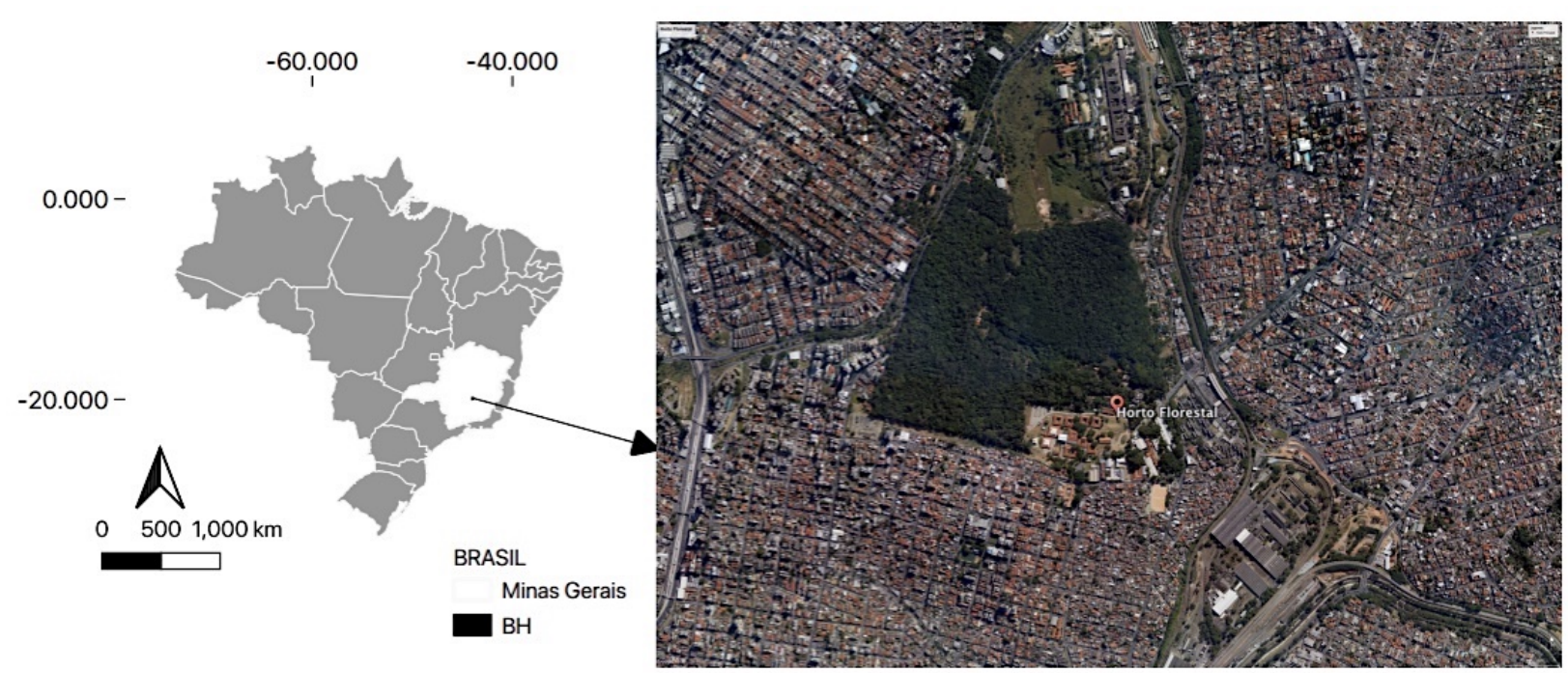

Fig. 1. Vista área da área do Museu de História Natural e Jardim Botânico da UFMG cuja entrada principal está no Bairro Santa Inês (Portaria 1), Belo Horizonte, Minas Gerais, Brasil. Fonte: Google Maps e RStudio.

Fig. 1. Aerial view of the UFMG Museum of Natural History and Botanical Garden area whose main entrance is in Bairro Santa Inês (Portaria 1), Belo Horizonte, Minas Gerais, Brazil. Source: Google Maps and RStudio. 


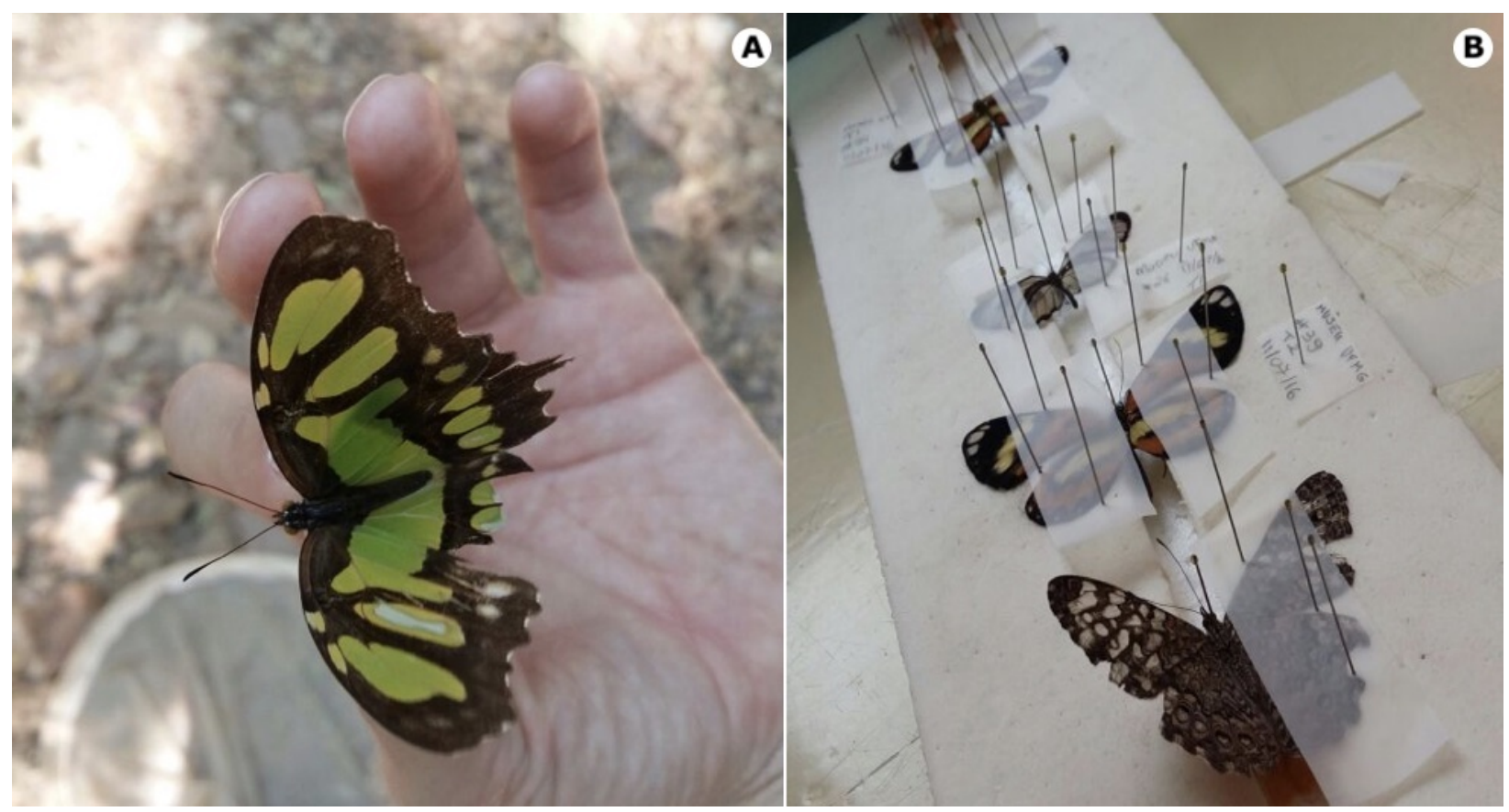

Fig. 2. A. Captura de Siproeta stelenes meridionales (Fruhstorfer, 1909) para posterior compressão torácica. B. Montagem das borboletas com esticador, papel vegetal e alfinete para secagem e ampliação da coleção do MNHJB-UFMG, Belo Horizonte, Minas Gerais, Brasil.

Fig. 2. A. Capture of Siproeta stelenes meridionales (Fruhstorfer, 1909) for later chest compression. $\mathrm{B}$. Assembly of the butterflies with stretchers, tracing paper and a pin for drying and expanding the MNHJB-UFMG collection, Belo Horizonte, Minas Gerais, Brazil.

cada espécie e as de difícil identificação foram eutanasiadas, por meio de compressão torácica, e armazenadas em envelopes entomológicos identificados. Em seguida foram montadas (Fig. 2B) e secas por uma hora em estufa a $50{ }^{\circ} \mathrm{C}$ e mantidas no laboratório de Zoologia do Centro Universitário UNA na coleção científica da instituição de ensino. Para identificação foi usado o site Buterflies of América (2017).

\section{ANÁLISE dos DADOS}

Para analisar o acumulo de espécies e a cobertura amostral, foram realizadas curvas de interpolação e extrapolação usando o pacote iNEXT (Hsieh et al., 2016) do programa R. São geradas três curvas, a primeira de acumulação de espécies, com o número de indivíduos no eixo $x$ e o número de espécies no $y$; a segunda de cobertura amostral (eixo y) pelo número de indivíduos (eixo $x$ ) e a terceira da riqueza (eixo y) pela cobertura amostral (eixo $x$ ). Neste trabalho, foram estabelecidas análises comparativas de riqueza, abundância entre meses e estações de coleta. Considerou-se época seca os meses de maio, julho e setembro e a época chuvosa os meses de janeiro, março e novembro como esperado para regiões tropicais.

Para os testes, usou-se o programa $\mathrm{R}$
Studio (R Core Team, 2017). Foram montados quatro modelos lineares generalizados, um com abundância e riqueza como variáveis respostas e meses e estações como variáveis explicativas. Utilizamos a quasipoisson como a distribuição de erros. Para testar se a composição de espécies muda entre os meses e entre as estações, foram realizadas duas análises permutacionais de variância, uma comparando a composição entre os meses e outra entre as estações. Em caso de significância, foi realizado um escalonamento multidimensional não métrico para a visualização das diferenças. E para testar cada escala de contribuições para a diversidade, fizemos uma partição aditiva da diversidade, onde a é a riqueza por mês de coleta, $\beta 1$ entre os meses de coleta e $\beta 2$ entre as estações do ano e o y é o total da riqueza amostrada. Para isso fizemos 100 randomizações para comparar os dados observados com os esperados. Essas análises foram realizadas com 0 pacote vegan (Oksanen et al., 2019). Para decomposição do $\beta$ em substituição de espécies ( $\beta$ sim) e aninhamento ( $\beta S N E$ ) utilizamos o pacote betapart (Baselga et al., 2020). Os gráficos foram todos feitos no Programa R Studio, com uso do pacote de dados ggplot2 (Wickham, 2016). 


\section{Resultados e discussão}

Foram coletados 571 indivíduos pertencentes a 95 espécies. A família mais abundantes foi Nymphalidae com 334 indivíduos de 37 espécies enquanto que Papilionidea, com apenas 5 indivíduos de 1 espécie, foi a menos abundante. Considerando a ocorrência de espécies por es- tação seca e chuvosa, foram encontradas 62 espécies e 263 indivíduos capturados na estação seca e 63 espécies e 308 indivíduos coletados na estação considerada chuvosa. Além disso, foram coletadas 31 espécies exclusivamente na estação seca e 32 espécies foram capturadas somente na estação chuvosa (Tab. 1). 44 espécies tiveram o registro de apenas um indivíduo

Tab. 1. Lista de espécies e respectivas abundâncias registradas na mata do Museu de História Natural e Jardim Botânico da UFMG entre junho de 2016 a maio de 2017, Belo Horizonte, Minas Gerais, Brasil. Número em negrito indica a riqueza por família ou subfamília. Os meses em azul representam o período chuvoso (janeiro, março e novembro) e os meses em laranja (maio, julho e setembro) a época seca.

Tab. 1. List of species and respective abundances recorded in the woods of the UFMG Natural History Museum and Botanical Garden between June 2016 to May 2017, Belo Horizonte, Minas Gerais, Brazil. Number in bold indicates wealth by family or subfamily. The blue months represent the rainy season (January, March and November) and the orange months (May, July and September) the dry season.

\begin{tabular}{|c|c|c|c|c|c|c|c|c|c|}
\hline \multirow{2}{*}{ Família / Subfamília / Espécie } & \multirow{2}{*}{ Abundância } & \multicolumn{2}{|c|}{ Estação } & \multicolumn{6}{|c|}{ Meses } \\
\hline & & Seca & Chuvosa & J & $\mathbf{M}$ & M & J & $\mathbf{s}$ & $\mathbf{N}$ \\
\hline \multicolumn{10}{|l|}{ Nymphalidae } \\
\hline \multicolumn{10}{|l|}{ Libytheinae } \\
\hline $\begin{array}{l}\text { Libytheana carinenta carinenta (Cramer, } \\
\text { 1777) }\end{array}$ & 9 & $\mathrm{x}$ & $\mathrm{x}$ & $\mathrm{x}$ & $\mathrm{x}$ & & $\mathrm{x}$ & $\mathrm{x}$ & \\
\hline \multicolumn{10}{|l|}{ Danainae } \\
\hline Aeria olena olena (Weymer, 1875) & 30 & $\mathrm{x}$ & $x$ & $\mathrm{x}$ & $\mathrm{x}$ & $x$ & $\mathrm{x}$ & & \\
\hline $\begin{array}{l}\text { Dircenna dero rhoeo (C. Felder \& R. } \\
\text { Felder, 1860) }\end{array}$ & 1 & & $\mathrm{x}$ & $\mathrm{x}$ & & & & & \\
\hline $\begin{array}{l}\text { Hypoleria lavinia lavinia (Hewitson, } \\
\text { 1855) }\end{array}$ & 1 & & $\mathrm{x}$ & & $\mathrm{x}$ & & & & \\
\hline Hypothyris euclea (Godart, 1819) & 6 & $x$ & $x$ & & & $\mathrm{x}$ & $x$ & & $\mathrm{x}$ \\
\hline $\begin{array}{l}\text { Ithomia agnosia zikani (R.F. d'Almeida, } \\
\text { 1940) }\end{array}$ & 4 & $\mathrm{x}$ & $\mathrm{x}$ & & $\mathrm{x}$ & & $\mathrm{x}$ & & \\
\hline Mcclungia cymo (Hübner, 1806) & 6 & & $\mathrm{x}$ & $\mathrm{x}$ & $\mathrm{x}$ & & & & $x$ \\
\hline $\begin{array}{l}\text { Mechanitis lysimnia lysimnia (Fabricius, } \\
\text { 1793) }\end{array}$ & 1 & $\mathrm{x}$ & & & & & $\mathrm{x}$ & & \\
\hline $\begin{array}{l}\text { Mechanitis polymnia casabranca } \\
\text { (Haensch, 1905) }\end{array}$ & 26 & $\mathrm{x}$ & $x$ & $\mathrm{x}$ & $\mathrm{x}$ & $\mathrm{x}$ & $\mathrm{x}$ & & $\mathrm{x}$ \\
\hline $\begin{array}{l}\text { Placidina euryanassa (C. Felder \& R. } \\
\text { Felder, 1860) }\end{array}$ & 1 & $x$ & & & & & $x$ & & \\
\hline \multicolumn{10}{|l|}{ Heliconiinae } \\
\hline Actinote parapheles (Jordan, 1913) & 2 & & $\mathrm{x}$ & & $\mathrm{x}$ & & & & $\mathrm{x}$ \\
\hline Actinote sp. 1 (Hübner, 1819) & 3 & & $x$ & & $\mathrm{x}$ & & & & $\mathrm{x}$ \\
\hline $\begin{array}{l}\text { Agraulis vanillae maculosa (Stichel, } \\
\text { 1908) }\end{array}$ & 4 & $\mathrm{x}$ & $x$ & $\mathrm{x}$ & $x$ & $x$ & & & \\
\hline
\end{tabular}


... continuação Tab. 1.

\begin{tabular}{|c|c|c|c|c|c|c|c|c|c|}
\hline Dryas iulia alcionea (Cramer, 1779) & 4 & $\mathrm{x}$ & $\mathrm{x}$ & & $x$ & & $x$ & & \\
\hline $\begin{array}{l}\text { Euptoieta hegesia meridiania (Stichel, } \\
\text { 1938) }\end{array}$ & 8 & & $\mathrm{x}$ & $\mathrm{x}$ & $\mathrm{x}$ & & & & $\mathrm{x}$ \\
\hline $\begin{array}{l}\text { Heliconius erato phyllis (Fabricius, } \\
1775 \text { ) }\end{array}$ & 45 & $x$ & $\mathrm{x}$ & $x$ & $\mathrm{x}$ & $\mathrm{x}$ & $\mathrm{x}$ & $\mathrm{x}$ & $x$ \\
\hline $\begin{array}{l}\text { Heliconius ethilla narcaea (Godart, } \\
\text { 1819) }\end{array}$ & 1 & & $\mathrm{x}$ & & & & & & $\mathrm{x}$ \\
\hline \multicolumn{10}{|l|}{ Limenitidinae } \\
\hline Adelpha sp. 1 (Hübner, 1819) & 1 & $x$ & & & & $\mathrm{x}$ & & & \\
\hline \multicolumn{10}{|l|}{ Biblidinae } \\
\hline $\begin{array}{l}\text { Biblis hyperia nectanabis (Fruhstorfer, } \\
\text { 1909) }\end{array}$ & 2 & $\mathrm{x}$ & $\mathrm{x}$ & & $\mathrm{x}$ & $\mathrm{x}$ & & & \\
\hline Ectima thecla thecla (Fabricius, 1796) & 1 & $\mathrm{x}$ & & & & & $\mathrm{x}$ & & \\
\hline $\begin{array}{l}\text { Hamadryas amphinome amphinome } \\
\text { (Linnaeus, 1767) }\end{array}$ & 5 & $\mathrm{x}$ & & & & & $\mathrm{x}$ & $x$ & \\
\hline Hamadryas arete (E. Doubleday, 1847) & 2 & $\mathrm{x}$ & & & & & & $\mathrm{x}$ & \\
\hline $\begin{array}{l}\text { Hamadryas februa februa (Hübner, } \\
\text { 1823) }\end{array}$ & 2 & $\mathrm{x}$ & & & & & $\mathrm{x}$ & $x$ & \\
\hline $\begin{array}{l}\text { Hamadryas feronia feronia (Linnaeus, } \\
\text { 1758) }\end{array}$ & 1 & $\mathrm{x}$ & & & & & $\mathrm{x}$ & & \\
\hline $\begin{array}{l}\text { Hamadryas iphthime iphthime (H. } \\
\text { Bates, 1864) }\end{array}$ & 1 & $\mathrm{x}$ & & & & & & $x$ & \\
\hline Hamadryas laodamia (Cramer, 1777) & 2 & $\mathrm{x}$ & & & & & & $\mathrm{x}$ & \\
\hline Nica flavilla flavilla (Godart, 1824) & 3 & $\mathrm{x}$ & $\mathrm{x}$ & & $\mathrm{x}$ & $\mathrm{x}$ & $\mathrm{x}$ & & \\
\hline \multicolumn{10}{|l|}{ Nymphalinae } \\
\hline Colobura dirce dirce (Linnaeus, 1758) & 3 & $\mathrm{x}$ & & & & & $\mathrm{x}$ & $\mathrm{x}$ & \\
\hline Ortilia ithra (W. F. Kirby, 1900) & 17 & $x$ & $\mathrm{x}$ & $\mathrm{x}$ & $\mathrm{x}$ & $\mathrm{x}$ & $\mathrm{x}$ & $\mathrm{x}$ & $x$ \\
\hline $\begin{array}{l}\text { Siproeta stelenes meridionalis } \\
\text { (Fruhstorfer, 1909) }\end{array}$ & 4 & $\mathrm{X}$ & $\mathrm{x}$ & $\mathrm{x}$ & & & $\mathrm{x}$ & $\mathrm{x}$ & \\
\hline Tegosa claudina (Eschscholtz, 1821) & 13 & $\mathrm{x}$ & $\mathrm{x}$ & & $\mathrm{x}$ & $\mathrm{x}$ & $x$ & $x$ & $\mathrm{x}$ \\
\hline \multicolumn{10}{|l|}{ Charaxinae } \\
\hline $\begin{array}{l}\text { Archaeoprepona demophon thalpius } \\
\text { (Hübner, 1814) }\end{array}$ & 1 & & $\mathrm{x}$ & $\mathrm{x}$ & & & & & \\
\hline $\begin{array}{l}\text { Memphis moruus stheno (Prittwitz, } \\
\text { 1865) }\end{array}$ & 1 & $\mathrm{x}$ & & & & & & $x$ & \\
\hline \multicolumn{10}{|l|}{ Satyrinae } \\
\hline $\begin{array}{l}\text { Catoblepia berecynthia unditaenia } \\
\text { (Fruhstorfer, 1907) }\end{array}$ & 1 & & $\mathrm{x}$ & & & & & & $x$ \\
\hline Hermeuptychia sp. 1 (Forster, 1964) & 43 & $x$ & $x$ & $x$ & $x$ & $x$ & $x$ & $x$ & $x$ \\
\hline
\end{tabular}

Rev. Biol. Neotrop. / J. Neotrop. Biol., Goiânia, v. 18, n. 1, p. 1-16, jan.-jun. 2021 
... continuação Tab. 1.

\begin{tabular}{|c|c|c|c|c|c|c|c|c|c|}
\hline Morpho menelaus (Linnaeus, 1758) & 2 & & $x$ & $x$ & & & & & \\
\hline Paryphthimoides eous (A. Butler, 1867) & 85 & $\mathrm{x}$ & $x$ & $x$ & $x$ & $\mathrm{x}$ & $\mathrm{x}$ & $\mathrm{x}$ & $x$ \\
\hline \multicolumn{10}{|l|}{ Hesperiidae } \\
\hline \multicolumn{10}{|l|}{ Eudaminae } \\
\hline Achlyodes busirus rioja (Evans, 1953 ) & 1 & & $x$ & & $\mathrm{x}$ & & & & \\
\hline $\begin{array}{l}\text { Aguna asander asander (Hewitson, } \\
\text { 1867) }\end{array}$ & 1 & $\mathrm{x}$ & & & & & & $\mathrm{x}$ & \\
\hline Aguna cirrus (Evans, 1952) & 1 & & $x$ & $x$ & & & & & \\
\hline Astrapes fulgerator (Walch, 1775) & 1 & $\mathrm{x}$ & & & & & $x$ & & \\
\hline $\begin{array}{l}\text { Epargyreus socus pseudexadeus } \\
\text { (Westwood, 1852) }\end{array}$ & 2 & $\mathrm{x}$ & $x$ & & $\mathrm{x}$ & & & $\mathrm{x}$ & \\
\hline $\begin{array}{l}\text { Phocides pigmalion hewitsonius } \\
\text { (Mabille, 1883) }\end{array}$ & 1 & & $x$ & & & & & & $\mathrm{x}$ \\
\hline Urbanus albimargo rica (Evans, 1952) & 1 & & $x$ & & $\mathrm{x}$ & & & & \\
\hline $\begin{array}{l}\text { Urbanus dorantes dorantes (Stoll, } \\
\text { 1790) }\end{array}$ & 4 & & $x$ & $x$ & $\mathrm{x}$ & & & & \\
\hline Urbanus procne (Plötz, 1881) & 1 & $\mathrm{x}$ & & & & & $x$ & & \\
\hline $\begin{array}{l}\text { Urbanus proteus proteus (Linnaeus, } \\
1758 \text { ) }\end{array}$ & 8 & $\mathrm{x}$ & $x$ & $x$ & $x$ & $x$ & & $x$ & $x$ \\
\hline
\end{tabular}

7 Pyrginae

Gorgythion begga begga (Prittwitz, 1868)

Heliopetes alana (Reakirt, 1868)

17

$x \quad x$

$x \quad x \quad x \quad x$

Heliopyrgus domicella willi (Plötz, 1884)

3

$x$

$x$

$\mathrm{x} x$

Pyrgus orcus (Stoll, 1780)

1

1

$x$

$\mathrm{x}$

Pythonides jovianus fabricii (W. F. Kirby, 1871)

6

Quadrus cerialis (Stoll, 1782)

2

Sostrata cronion (C. Felder \& R. Felder, 1867)

Staphylus ascalan (Staudinger, 1876)

Theagenes dichrous (Mabille, 1878)

Trina geometrina geometrina (C. Felder \& R. Felder, 1867)

Xenophanes tryxus (Stoll, 1780)

1

$x \quad x$

$\mathrm{x}$

Hesperiinae

Anthoptus epictetus (Fabricius, 1793)

\begin{tabular}{lllllllll}
5 & $x$ & & & $x$ & $x$ & & $x$ & $x$ \\
2 & $x$ & $x$ & & $x$ & $x$ & & & \\
8 & $x$ & $x$ & $x$ & $x$ & & & $x$ & $x$ \\
\hline
\end{tabular}

Arotis sp. 1 (Mabille, 1904)

Cumbre cumbre (Schaus, 1902)

$\mathrm{x} \quad \mathrm{X}$

2

2

$\mathrm{X}$

$\mathrm{x}$

$3-x$

$x$

$\mathrm{x}$

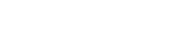


... continuação Tab. 1.

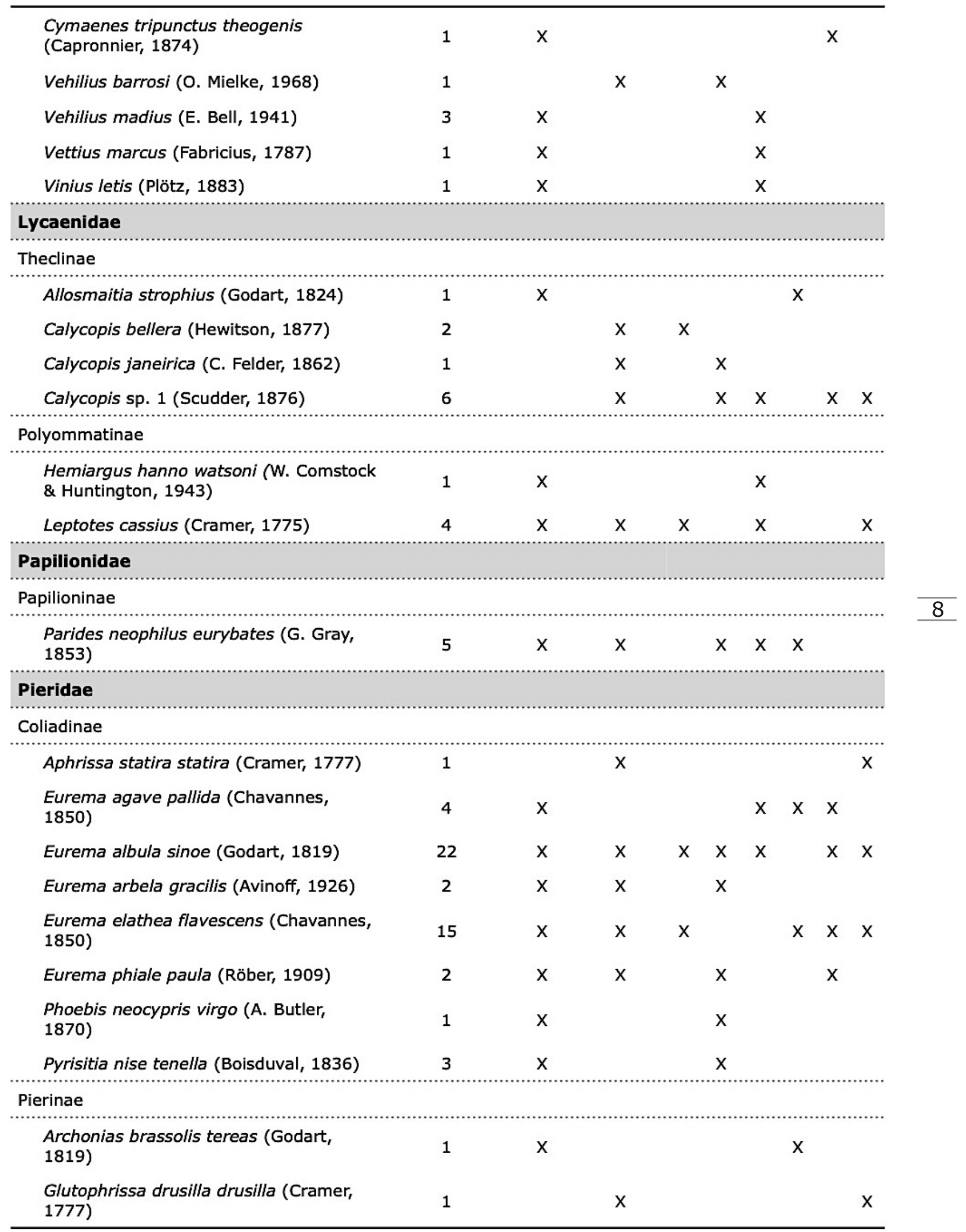


... continuação Tab. 1.

Riodinidae

Riodininae

Charis anius (Cramer, 1776)

1

$\mathrm{X}$

$\mathrm{X}$

Detritivora sp.1 (J. Hall \& Harvey, 2002)

40

$\mathrm{X}$

$x$

Eurybia pergaea (Geyer, 1832)

3

$\mathrm{X}$

$\mathrm{X} \quad \mathrm{x}$

Leucochimona icare matatha (Hewitson, 1873)

Melanis xenia xenia (Hewitson, 1853)

Panara soana bacana (Callaghan, 1997)

Perophthalma tullius (Fabricius, 1787)

Stalachtis phlegia phlegetontia (Perty, 1833)

$1 \quad x$

$\mathrm{X}$

$1 \quad x$

$4 \quad x \quad x$

$1 \quad \mathrm{X}$

$x \quad x \quad x$

Synargis calyce (C. Felder \& R. Felder, 1862)

1

$x$

$\mathrm{X}$

Synargis paulistina (Stichel, 1910)

18

X $\quad x$

$\mathrm{X} \times \mathrm{X}$

1

$x \quad x$

Sem identificação

Hesperiidae sp. 1

1

3

$\mathrm{X}$

Riodinidae sp. 1

$\mathrm{x}$

$\mathrm{X}$

$\mathrm{X}$

(singletons) e 14 espécies tiveram o registro de dois indivíduos (doubletons). Não foi observado a assíntota da curva (Fig. 3A), mas a cobertura amostral foi aproximadamente 93\% (Fig. 3B e 3C). A abundância e a riqueza não variaram entre os meses $(F=1,227, p=0,337 ; F=1,134$, $\mathrm{p}=0,378$, respectivamente) e nem entre as estações ( $F=0,7749, p=0,388 ; F=1,0045, p$ $=0,327$, respectivamente). Já a composição das espécies diferiu entre os meses $(p=0,003$; Fig. 4A) e entre as estações ( $p=0,017$; Fig. $4 B$ ). A escala de diversidade que mais contribuiu pra riqueza foi o $\beta 2$ (cerca de $50 \%$ do total da diversidade), confirmando que a maior diferença entre está entre as estações (Fig. 5). Comparando os resultados dos dados esperados e observados, o a, a diversidade no mês, foi maior que a esperada e o $\beta 2$, a diferença entre os meses menor que o esperado. A substituição de espécies foi o mecanismo que mais contribuiu com o beta temporal ( $\beta$ sim $=0.91 ; \beta S N E$ $=0.02$ ).

Ao longo de todo o estudo, foram coletadas, marcadas e soltas 32 borboletas por serem de fácil identificação. Destas, apenas dois indivíduos de Heliconius erato phyllis (Fabricius, 1775) foram recapturadas e, em seguida, soltas. Uma delas sobreviveu por três meses e foi recapturada na mesma área em que foi primari- amente coletado. Já o outro indivíduo foi recapturado no mesmo dia e pode ter percorrido até quatro quilômetros entre a trilha três e a trilha quatro, onde foi novamente recapturado (Tab. 2).

Em regiões temperadas, a variação sazonal dos insetos está diretamente relacionada com a temperatura e a disponibilidade de luz. Já nas zonas tropicais, a precipitação e a umidade também são fatores importantes para definir as flutuações desses organismos ao longo do ano (Hamer et al., 2005).

A mudança das condições climáticas durante as estações afeta a disponibilidade de recursos bióticos e abióticos e pode ser determinante para dinâmica das comunidades dos insetos (Wagner 2003, Grotan et al., 2012). Em se tratando da diversidade de borboletas frugívoras, a quantidade de chuvas tem sido um fator capaz de explicar a variação nesse padrão (DeVries et al., 2001). De modo geral, em ambientes não antropizados, espera-se maior diversidade e número de indivíduos no período chuvoso (meses mais úmidos e quentes), quando há mais recursos alimentares disponíveis para o consumo das borboletas, assim como as condições que favoreçam a atividade reprodutiva e a eclosão de ovos e pupas (Devries et al., 1997). 

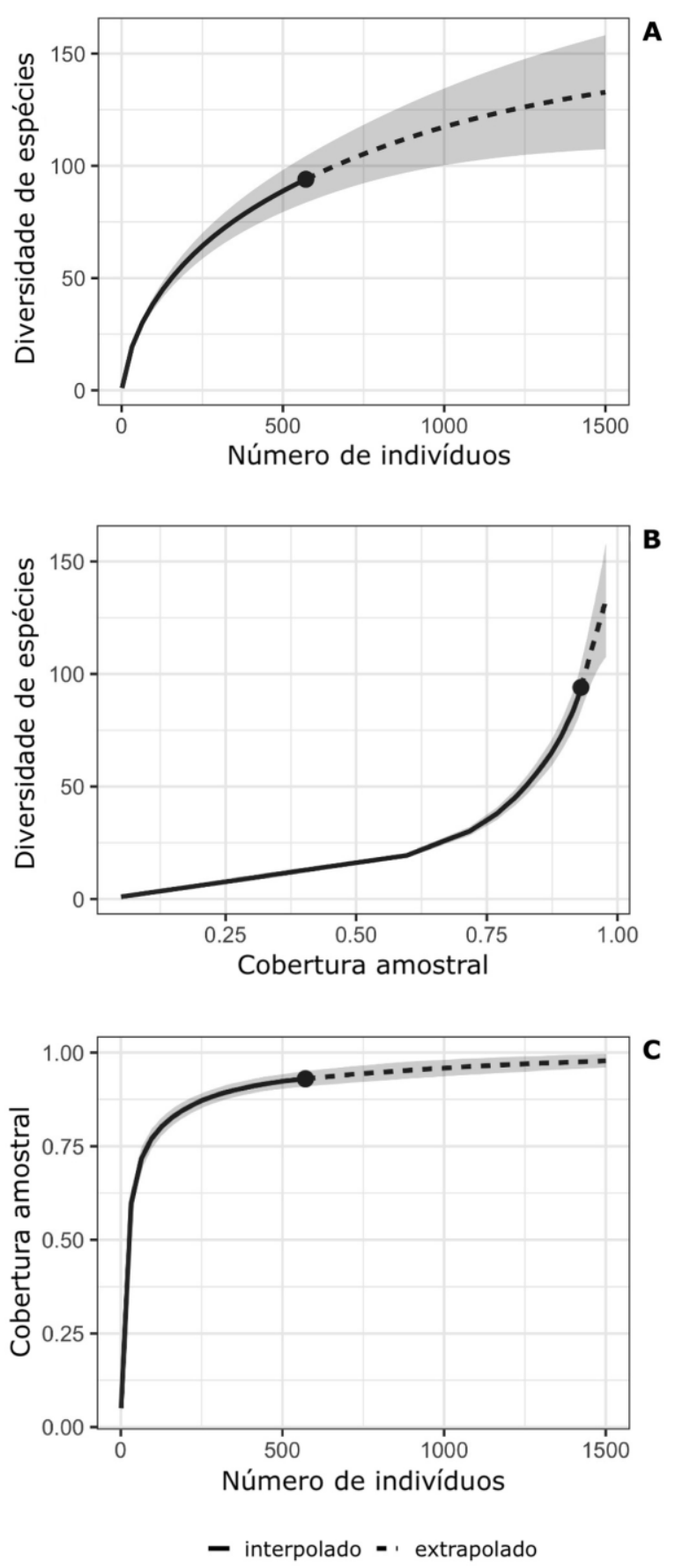

Fig. 3. A. Curva de acúmulo de espécies de borboletas registradas na mata do MHNJB-UFMG no período de junho de 2016 a maio de 2017, Belo Horizonte, Minas Gerais, Brasil. B. Curva de cobertura amostral. C. Curva de riqueza.

Fig. 3. A. Accumulation curve of butterfly species recorded in the woods of the MHNJB-UFMG from June 2016 to May 2017, Belo Horizonte, Minas Gerais, Brazil. B. Sample coverage curve. C. Richness curve.
Entretanto, em segmentos florestais urbanos, esse mesmo padrão não é tão bem descrito. E, embora já tenham sido feitos alguns trabalhos caracterizando a assembleia de borboletas nas matas de Belo Horizonte e região metropolitana (Brown Jr. \& Mielke, 1968; Brown Jr. \& Freitas, 2000; Silva et al., 2007; Silva et al., 2012; Soares et al., 2012), poucos são os que relacionaram sazonalidade com riqueza, abundância e composição.

Na Área de Proteção Especial Manancial Cercadinho, foram identificados 170 indivíduos pertencentes a 91 espécies de borboletas com uso de armadilhas entomológicas (Silva et al., 2007) e a maior abundância coincidiu com os meses mais quentes e de moderada para alta precipitação. Em 2014, foi realizado, também na mata do MHNJB-UFMG, um trabalho cujo objetivo foi fazer um levantamento apenas das espécies de borboletas frugívoras, comparando as estações secas e chuvosas (Silva et al., 2014). $\mathrm{Na}$ estação chuvosa houve maior riqueza e a estação seca destacou-se por maior abundância.

Utilizando a metodologia de armadilhas foram coletados 818 indivíduos de 24 espécies. Entre elas, 13 foram exclusivas do estudo e 11 espécies são comuns com o atual trabalho. Assim, somando-se os dois trabalhos, o número total de espécies de borboletas do Museu da UFMG é de pelo menos 109, já que a curva de acumulação das espécies capturadas não se estabilizou.

No presente estudo, a riqueza e a abundância não diferiram no âmbito geral entre as estações secas e chuvosas. Os mesmos resultados podem não ter sido encontrados, possivelmente em função de variações nos níveis de precipitação encontrados na época seca durante o estudo. Nos meses de junho e setembro de 2016, que são caracterizados como épocas secas, foram registradas chuvas acima da média e nos meses considerados chuvosos, janeiro e fevereiro de 2017, foram observadas chuvas abaixo da média para a capital mineira (Climatempo, 2017).

A dificuldade em entender os padrões temporais em tais ecossistemas é justificada pela atividade humana que causou alterações significativas na distribuição e fisionomia destes habitats, e na dinâmica das populações que vivem nessas áreas fragmentadas, incluindo a das borboletas. Assim, um conjunto de fatores, tais como microclima da região, heterogeneidade do espaço, isolamento e tamanho dos fragmentos, recursos alimentares presentes, também pode influenciar a sazonalidade das espécies e explicar seu padrão de distribuição em parques urbanos (Ruszczyk, 1986; Stefanesco et al., 2004). A maior parte do Museu é composta por matas, mas também há áreas abertas, trilhas, um grande lago, uma nascente e jardins artifici- 

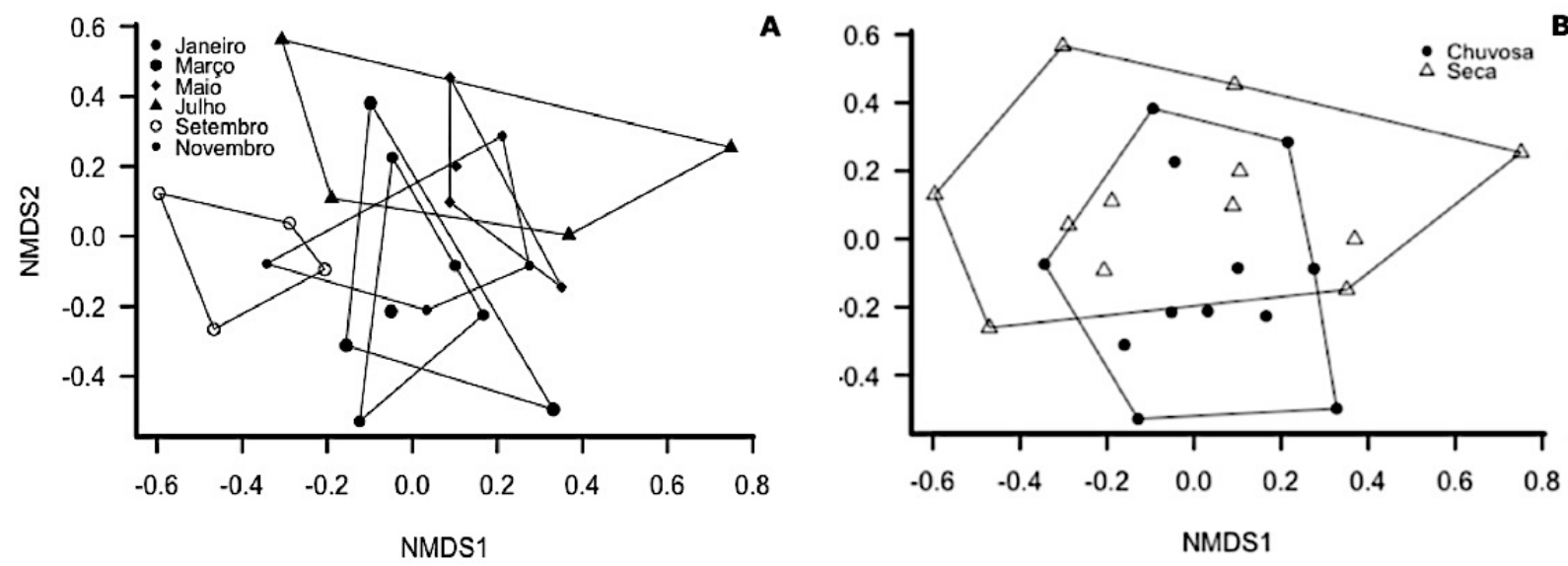

Fig. 4. A. Variação da composição entre os meses de coleta no MHNJB-UFMG, Belo Horizonte, Minas Gerais, Brasil. B. Variação da composição entre as estações do ano para a coleta. NMDS: escalonamento multidimensional não métrico.

Fig. 4. A. Composition variation between collection months in the MHNJB-UFMG, Belo Horizonte, Minas Gerais, Brazil. B. Composition variation between collection seasons of the year. NMDS: nonmetric multidimensional scaling .

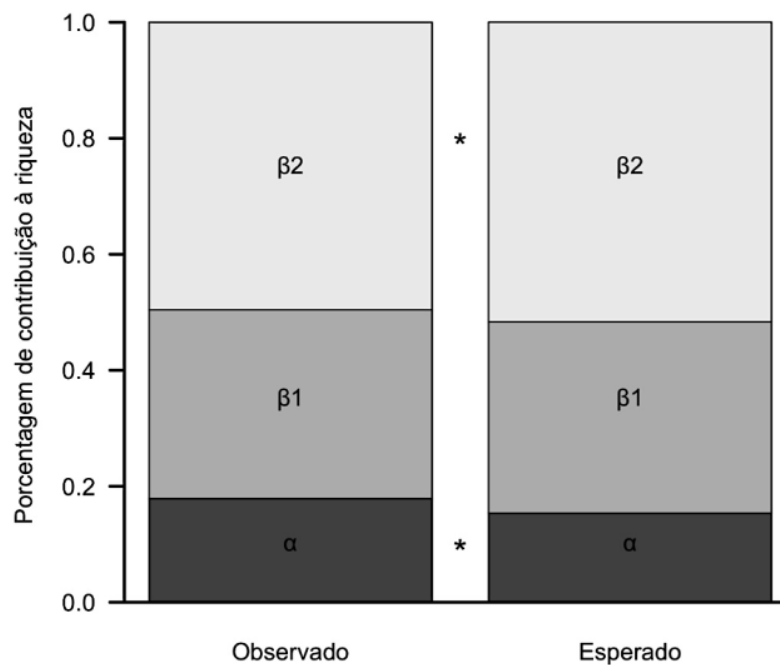

Fig. 5. Diversidade alfa, beta e gama. a é a riqueza por mês de coleta, $\beta 1$ entre os meses de coleta e $\beta 2$ entre as estações do ano e o y é o total da riqueza amostrada.

Fig. 5. Alpha, beta and gamma diversity. $a$ is the richness per month of collection, $\beta 1$ between the months of collection and $\beta 2$ between the seasons of the year and $Y$ is the total richness sampled.

ais. Isso favorece a ocupação do espaço de maneiras diferentes e, junto com a pluviosidade, também podem explicar a ausência na variação da riqueza e abundância de modo geral.

O presente trabalho apresentou maior ri- queza que os citados acima, em Belo Horizonte, possivelmente pela diferença do tamanho das áreas de coleta. A mata da PUC-MG, bairro Coração Eucarístico, possui uma área de $70 \mathrm{mil}$ metros quadrados e o Parque Municipal, que fica no centro da cidade, abrange uma área total de 82 mil metros quadrados (Prefeitura de Belo Horizonte, 2017), enquanto a área do Museu é de, aproximadamente, $600 \mathrm{mil} \mathrm{m}^{2}$. No caso do Parque Municipal, a diferença ainda se deve à localização em região central da cidade: de acordo com Ruszczyk (1986), o grau de urbanização é inversamente proporcional à riqueza. Em Nova Lima, embora a área seja mais bem preservada e expressivamente maior, 1.500 .000 $\mathrm{m}^{2}$, a metodologia empregada foi apenas a de armadilhas para borboletas frugívoras e, por isso, apresentou menor diversidade.

A composição variou de acordo com os meses e as estações. Com isso, é possível prever a época do ano em que as espécies ocorrerão como mostrado na Tab. 1.

A maior abundancia e riqueza de Nymphalidae corrobora com o fato de que cerca de $30 \%$ do total de borboletas de uma área de ambiente neotropical são pertencentes à essa família (Brown Jr. \& Freitas, 2000). Esta grande variação é consequência da família possuir maior número de espécies, associações a um maior número de plantas hospedeiras e ao fato de suas larvas possuírem maior diversidade de formas (Duarte et al., 2012).

As espécies encontradas durante a maior parte do ano são generalistas ou bem adaptadas a ambientes modificados pelo homem (Canals, 2000). Satiríneos (Nymphalidae) foram 
Tab. 2. Espécies recapturadas e soltas na mata do MNHJB-UFMG, Belo Horizonte, Minas Gerais, Brasil.

Tab. 2. Species recaptured and released in the woods of MNHJB-UFMG, Belo Horizonte, Minas Gerais, Brazil.

\begin{tabular}{ccccc}
\hline Espécie & Data da coleta & $\begin{array}{c}\text { Data da } \\
\text { recaptura }\end{array}$ & $\begin{array}{c}\text { Tempo de } \\
\text { recaptura }\end{array}$ & $\begin{array}{c}\text { Distância } \\
\text { percorrida }\end{array}$ \\
\hline $\begin{array}{c}\text { Heliconlus erato phyllis } \\
\text { (Fabricius, 1775, } \\
\text { Nymphalidae) }\end{array}$ & $07 / 01 / 2017$ & $11 / 03 / 2017$ & 4 dias & 0 (permaneceu na \\
Área)
\end{tabular}

mais presentes na época chuvosa. Esse período corresponde ao crescimento das gramíneas que são o tipo de planta mais usada como hospedeira pelas larvas (Duarte et al., 2012).

De hábitos mais generalistas, os hesperídios também figuram entre as famílias mais diversas junto com os nimphalídeos. Podem ocorrer tanto em ambientes mais abertos quanto nos fechados, sendo muitas espécies atraídas por fezes de pássaros (Lamas et al., 2004; Bonfantti et al., 2009).

Os riodinídeos, por sua vez, apresentam preferência por ambientes florestais, embora alguns gêneros, como Charis, prefiram áreas abertas e ensolaradas (DeVries, 2001). A alimentação é variada: os gêneros Leucochimona e Perophthalma se alimentam de Rubiaceae (DeVries, 1997), segunda família de plantas mais abundante no Museu (Felix, 2009). Eurybia se alimentam de Zingiberaceae (DeVries, 1997), representada por uma única espécie na área (Felix, 2009). Melanis se alimentam de Fabaceae impalatáveis (DeVries, 1997), que são a família de plantas mais comum no Museu (Felix, 2009). Já Synargis se alimentam de várias espécies de plantas, justificando sua presença nas duas estações (DeVries, 1997).

O fato de algumas espécies dessa família não terem sido notadas nas duas estações pode estar relacionado à época de floração das plantas das quais elas se alimentam no Museu e ao fato dos riodinideos apresentarem algumas particularidades, como voo em ambientes específicos, período de atividade limitado e comportamento esquivo, que dificultam sua real amostragem. Portanto, sua riqueza pode ser ainda maior na área amostrada e seu padrão sazonal mais diversificado (DeVries, 1997).

Já a família Pieridae, no geral, está mais associada às áreas abertas, uma das razões pela qual foram menos diversos, pois o Museu é predominantemente coberto por mata (Brown Jr. \& Freitas, 1999). O gênero Eurema, com cinco espécies, foi o único presente em todos os meses e é característico de áreas alteradas (Brown Jr., 1992).

Embora licenídeos sejam bem abundantes na região tropical, superando, muitas vezes, nimphalídeos e hesperídeos, são típicos de regiões mais abertas. Também são menos tolerantes à atividade antrópica, além de manterem especializações com plantas hospedeiras, motivo pelo qual podem ter sido encontradas em épocas definidas e em menor riqueza do que a esperada para a família em regiões tropicais (Brown Jr. \& Freitas, 1999).

Por fim, mesmo que Papilionidae seja uma família bioindicadora da qualidade ambiental, alguns gêneros, como Parides, têm grande tolerância à perturbação humana (Devries, 1997). Porém, são típicas de áreas abertas e não de mata como no Museu. Seu principal alimento são as plantas da família Aristolochiaceae representadas por duas espécies no local de estudo e que são pouco abundantes em períodos frios e secos (Devries, 1997; Felix, 2009). Esses fatores podem justificar a pouca abundância dessa família e a sua sazonalidade marcante.

Com relação as espécies ameaçadas de extinção, nenhuma das capturadas consta no livro vermelho das espécies em extinção do ICMBIO. (ICMBio/MMA 2018). Entretando, houve registro de espécies raras assim como nos estudos de Brown Jr. (1992); Brown Jr. \& Freitas (2000). São elas: Aguna asander asander (Hewitson, 1867), Hesperiidae; Archonias brassolis tereas (Godart, 1819), Pieridae; Hamadryas arete (E. Doubleday, 1847), Hamadryas feronia feronia (Linnaeus, 1758), Hypoleria lavinia lavinia (Hewitson, 1855), Morpho menelaus (Linnaeus, 1758), Nymphalidae; Phocides 
pigmalion hewitsonius (Mabille, 1883) Vinius letis (Plötz, 1883), Hesperiidae, condizendo com os poucos indivíduos coletados dessas espécies no presente trabalho. Segundo Magurran (2011), é comum que a maior parte das espécies de uma comunidade seja rara e um pequeno número de espécies tende a representar a maior parte dos indivíduos amostrados.

No presente estudo, as espécies mais abundantes foram Aeria olena olena (Weymer, 1875), Heliconius erato phyllis (Fabricius, 1775), Hermeuptychia sp. 1 (Forster, 1964), Paryphthimoides eous (A. Butler, 1867) e Detritivora sp. 1 (J. Hall \& Harvey, 2002), Nymphalidae, comuns em ambientes urbanos e em matas secundárias (Canals, 2000).

No Museu, já foi feito um estudo da composição florista da área (Felix, 2009). Vale relacionar esse estudo com os hábitos alimentares das borboletas para elucidar, junto com a análise espacial, os demais fatores que interferem na distribuição desses lepidópteros no MNHJB, além da sazonalidade. Apesar de não tenha sido o foco da pesquisa, uma análise inicial mostrou maior abundância nas áreas abertas em relação às fechadas com a riqueza não se alterando entre os locais.

Os resultados encontrados evidenciam aspectos da ecologia de borboletas em áreas urbanas fragmentadas e auxiliam na tomada de decisões que favoreçam a preservação dessas espécies. Este estudo destaca a importância de uma pequena área, em um grande centro urbano, abrigar, no mínimo, 109 espécies de borboletas, incluindo espécies raras. Também enfatiza a importância das áreas verdes nas cidades como áreas relevantes para a proteção da biodiversidade local e fomento da educação ambiental de forma acessível a diferentes públicos. Um dos resultados desse trabalho foi a elaboração de um guia impresso "Borboletas do MHNJB - UFMG - Série caderno do educador ambiental - volume 2" (Silva et al., 2017) para o uso em atividades de educação ambiental, totalizando a distribuição de 25 cópias para o local.

\section{Agradecimentos}

Ao Centro Universitário UNA, por nos possibilitar a realização do projeto, assim como à Bióloga do MHNJB-UFMG, Flávia Santos Faria. Também merecem agradecimentos os alunos que participaram do projeto, Bryan Ôrtero, Larissa Pereira, Isabella Louise, e a fotógrafa Magê Monteiro.

\section{REFERÊNCIAS}

Andres B., D. Orme, S. Villeger, J. Bortoli, F. L. \& M. Logez. 2020. Betapart: Partitioning Beta Diversity into Turnover and
Nestedness Components. R package version 1.5.2. Disponível em: <https://CRAN.Rproject.org/package=betapart $>$. Acesso em 25 nov. 2020.

Beutel, R. G., F. Friedrich, S. Q. Ge \& X. K. Yang. 2014. Insect Morphology and Phylogeny. Berlin, Gruyter.

Boggs, C. L., W. B. Watt \& P. R. Ehrlich. 2003. Butterflies: ecology and evolution taking flight. Chicago, University of Chicago Press.

Bonfantti, D., R. A. Di Mare \& R. Giovenardi. 2009. Butterflies (Lepidoptera: Papilionoidea and Hesperioidea) from two forest fragments in northern Rio Grande do Sul, Brazil. Check List. 5: 819-829. DOI: https:// doi.org/10.15560/5.4.819

Breuste, J. H. \& M. Artmann. 2015. Allotment gardens contribute to urban ecosystem service: case study Salzburg, Austria. J. Urban Plan. Dev. 141(3): A5014005. DOI: https://doi.org/10.1061/(ASCE)UP.19435444.0000264

Brito, M. M., D. B. Ribeiro, M. Raniero, E. Hasui, F. N. Ramos \& A. Arab. 2014. Functional composition and phenology of fruit-feeding butterflies in a fragmented landscape: variation of seasonality between habitat specialists. J. Insect Conserv. 18(4): 547-560. DOI: https://doi.org/ 10.1007/s10841-014-9650-8

Brown Jr., K. S. \& O. H. H. Mielke. 1968. Lepidoptera of the Central Brazil Plateau. III. Partial list for the Belo Horizonte area, showing the character of the southeastern "Blend Zone". J. Lepid. Soc. 22(3): 147157.

Brown Jr., K. S. 1992. Borboletas da Serra do Japi: diversidade, habitats, recursos alimentares e variação temporal. pp. 142186. In: Patricia, L. \& C. Morellato (Org.). História Natural da Serra do Japi: Ecologia e preservação de uma área florestal no Sudeste do Brasil. Campinas, FAPESP.

Brown Jr., K. S. \& A. V. L. Freitas. 1999. Lepidoptera, pp. 225-243. In: Joly, C. A. \& C.E.M. Bicudo (Org.). Biodiversidade do Estado de São Paulo, Brasil: síntese do conhecimento ao final do século $X X$, Invertebrados Terrestres. São Paulo, FAPESP.

Brown Jr., K. S. \& A. V. L. Freitas. 2000. Diversidade de Lepidoptera em Santa Teresa, Espírito Santo. Bol. Mus. Biol. Mello Leitão. 11(12): 71-118. 
Brown Jr., K. S. \& A. V. L. Freitas. 2000. Atlantic forest butterflies: indicators for landscape conservation. Biotropica. 32 (4): 934-956. DOI: https://doi.org/10.1111/j. 1744-7429.2000.tb00631.x

Brown Jr., K. S. \& A. V. L. Freitas. 2002. Butterfly communities of urban forest fragments in Campinas, São Paulo, Brazil: structure, instability, environmental correlates, and conservation. J. Insect Conserv. 6: 217-231. DOI: https://doi.org/10.1023/ A: 1024462523826

Buterflies of América. 2017. Disponível em: <https://www.butterfliesofamerica.com>. Acesso em 05 de mai. 2019.

Canals, G. R. 2000. Butterflies of Buenos Aires. Buenos Aires, L.O.L.A.

Chan, K. M. A., P. Balvanera, K. Benessaiah, M. Chapman, S. Díaz, E. Gómez-Baggethun, R. Gould, N. Hannahs, K. Jax, S. Klain, G. Luck, B. Martín-López, B. Muraca, B. Norton, K. Ott, U. Pascual, T. Satterfield, M. Tadaki, J. Taggart \& N. Turner. 2016. Opinion: Why protect nature? Rethinking values and the environment. P. Natl. Acad. Sci. USA. 113(6): 1462-1465. DOI: https://doi.org/10.1073/ pnas. 1525002113

Climatempo. 2017. Portal Climatempo. BH acumula só um terço da chuva de 2017. Disponível em: <https://www.climatempo.com.br/noticia/2017/10/20/bh.acumula.so.um.terco.da.chuva.de.2017-1569>. Acesso 20 mai. 2017.

DeVries, P. J., D. Murray. \& E. R. Lande. 1997. Species diversity in vertical, horizontal, and temporal dimensions of a fruit-feeding butterfly community in an Ecuadorian rainforest. Biol. J. Linn. Soc., London. 62(3): 343-364.

DeVries, P. J. \& T. R. Walla. 2001. Species diversity and community structure in Neotropical fruit-feeding butterflies. Biol. J. Linn. Soc. 74(1): 1-15. DOI: https:// doi.org/10.1006/bijl.2001.0571

Duarte, M., G. Marconato, A. Specht \& M. Casagrande. 2012. Lepidoptera. pp. 625679. In: Rafael, J. A., G. A. R. Melo, C. J. B. Carvalho, S. A. Casari \& R. Constantino (Org.). Insetos do Brasil: Diversidade e Taxonomia. Ribeirão Preto, Holos.
Felix, D. F. 2009. Composição florística do Museu de História Natural e Jardim Botânico da Universidade Federal de Minas Gerais, Belo Horizonte, Minas Gerais. 111 f. Dissertação (Mestrado em Biologia Vegetal), Universidade Federal de Minas Gerais, Belo Horizonte, Minas Gerais.

Filgueiras, B. K. C., D. H. A. Melo, I. R. Leal, M. Tabarelli, A. V. L. Freitas \& L. Iannuzzi. 2016. Fruit-feeding butterflies in edge-dominated habitats: community structure, species persistence and cascade effect. J. Insect Conserv. 20: 539-548. DOI: https://doi.org/10.1007/s10841-0169888-4.

Freitas, A. V. F., C. A. Iserhard, J. P. Santos, J. Y. O. Carreira, D. B. Ribeiro, D. H. A. Melo, A. H. B. Rosa, O. J. MariniFilho, G. M. Acácio \& M. Uehara-Prado. 2014. Studies with butterfly bait traps: an overview. Rev. Colomb. Entomol. 40(2): 203-212.

Grotan, V., R. Lande, S. Engen, B. Sæther \& P. J. DeVries. 2012. Seasonal cycles of species diversity and similarity in a tropical butterfly community. J. Anim. Ecol. 81(3): 714-723. DOI: https://doi.org/ 10.1111/j. 1365-2656.2011.01950.x.

Hamer, K. C., J. K. Hill, N. Mustaffa, S. Benedick, T. N. Sherratt, V. K. Chey \& M. Maryati. 2005. Temporal variation in abundance and diversity of butterflies in Bornean rain forests: opposite impacts of logging recorded in different seasons. J. Trop. Ecol. 21(4): 417-425. DOI: https:// doi.org/10.1017/S026646705002361.

ICMBio/MMA. 2018. Livro Vermelho da Fauna Brasileira Ameaçada de Extinção: Volume VII - Invertebrados 1 ed. Brasília, ICMBio/ MMA. Disponível em: <https://www.icmbio.gov.br/portal/images/stories/comunicacao/publicacoes/publicacoes-diversas/livro _vermelho_2018_vol7.pdf>. Acesso em 22 out. 2020.

Jari O., F. G. Blanchet, M. Friendly, R. Kindt, P. Legendre, D. McGlinn, P. R. Minchin, R. B. O'Hara, G. L. Simpson, P. Solymos, M. Henry, H. Stevens, E. Szoecs \& H. Wagner. 2019. Vegan: Community Ecology Package. R package version 2.5-6. Disponível em: <https:// CRAN.R-project.org/package $=$ vegan $>$. Acesso em 24 nov. 2020. 
Lamas, G. 2004. Atlas of Tropical Lepidoptera: checklist. Assoc. Trop. Lepid. Scientific Publishers, Gainesville, 439 p.

Magurran, A. E. 2011. Medindo a Diversidade Biológica. Paraná. UFPR.

Oksanen, J., F. G. Blanchet, M. Friendly, R. Kindt, P. Legendre, D. McGlinn, P. R. Minchin, R. B. O'Hara, G. L. Simpson, P. Solymos, M. H. H. Stevens, E. Szoecs \& H. Wagner. 2019. Vegan - Community Ecology Package. R Package version 2. 5-6. Disponível em: <https://CRAN.R-project.org/package $=$ vegan $>$. Acesso em 18 nov. 2020.

Oliveira, M. A. D. E., C. F. F. Gomes, E. M. Pires, C. G. S. Marinho \& T. M. C. Della Lucia. 2014. Bioindicadores ambientais: insetos como um instrumento desta avaliação. Ver. Ceres. 61: 800-807 (suplemento). DOI: http://dx.doi.org/ 10.1590/0034-737X201461000005

Prefeitura de Belo Horizonte. 2017. Minas Gerais. Portal Prefeitura de Belo Horizonte. Disponível em: <http://www.belohorizonte.mg.gov.br/local/servico-turistico/espaco-para-evento/aberto/ parque-municipal-americo-renne-giannetti>. Acesso em 20 abr. 2017.

R Core Team. 2017. R: A language and environment for statistical computing. R Foundation for Statistical Computing, Vienna, Austria. Disponível em: <https://www.Rproject.org/>. Acesso em 25 jun. 2020.

Ribeiro, D. B., P. I. Prado, K. S. Brown Jr. \& A. V. L. Freitas. 2010. Temporal Diversity Patterns and Phenology in Fruit-feeding Butterflies in the Atlantic Forest. Biotropica. 42(6): 710-716. DOI: https://doi.org/ 10.1111/j.1744-7429.2010.00648.x.

Ricklefs, R. E. 2003. A Economia da Natureza. 5 ed. Rio de Janeiro, Guanabara Koogan.

Rocha, C. F. D., H. G. Bergallo, M. Van Sluys \& M. A. S. Alves. 2006. Biologia da Conservação: essências. São Carlos, Editora Rima.

Ruszczyk, A. 1986. Ecologia urbana de borboletas, I. O gradiente de urbanização e a fauna de Porto Alegre-RS. Ver. Bras. Bio. 46: 675-688.
Silva, A. R. M, E, V. D. Silva W. X. De Deus \& O. H. Costa. 2014. Borboletas frugívoras (Lepidoptera: Nymphalidae) da mata do Museu de História Natural e Jardim Botânico da UFMG, Belo Horizonte, Minas Gerais, Brasil. Arq. do Mus. de Hist. Nat. e J. Bot. 23: 33.

Silva, A. R. M, G. G. Landa \& R. F. Vitalino. 2007. Borboletas (Lepidoptera) de um fragmento de mata urbano em Minas Gerais, Brasil. Lundiana. 8(2): 137-142.

Silva, A. R. M., C. O. Castro, P. O. Mafia, M. O. C. Mendonca, T. C. C. Alves \& M. V. Beirão. 2012. Borboletas frugívoras (Lepidoptera: Nymphalidae) de uma área urbana (Area de Proteção Especial Manancial Cercadinho) em Belo Horizonte, Minas Gerais, Brasil. Biota Neotrop. 12: 292-297. DOI: $\quad$ https://doi.org/10.1590/S167606032012000300028.

Soares, G. R., A. P. Oliveira \& A. R. M. Silva. 2012. Borboletas (Lepidoptera: Papilionoidea e Hesperioidea) de um parque urbano em Belo Horizonte, Minas Gerais, Brasil. Biota Neotrop. 12: 209-217. DOI: https:// doi.org/10.1590/S167606032012000400022.

Soga, M., T. Kawahara, K. Fukuyama, K. Sayama, T. Kato, M. Shimomura, T. Itoh, T. Yoshida \& K. Ozaki. 2015. Landscape versus local factors shaping butterfly communities in fragmented landscapes: Does host plant diversity matter? J. Insect Conserv. 19: 781-790. DOI: https://doi.org/10.1007/s10841-015-97999

Stefanescu, C., S. Herrando \& F. Páramo. 2004. Butterfly species richness in the north-west Mediterranean Basin: the role of natural and human-induced factors. J. Biogeogr. 31: 905-915. DOI: https:// doi.org/10.1111/j.13652699.2004.01088.x

Vanin, S. A. 2012. Filogenia e Classificação. pp. 81-10. In: Rafael, J. A., G. A. R. Melo, C. J. B. Carvalho, S. A. Casari \& R. Constantino. Insetos do Brasil. Diversidade e Taxonomia. 1 ed. Ribeirão Preto, Holos.

Verzoça, F. C. \& R. F. Bion. 2011. Polinização de Lantana fucata Lindley (Verbenaceae) por Parides ascanius Cramer (Lepidoptera: Papilionoidea) na Restinga de Grumari, Rio de Janeiro. EntomoBrasilis. 4(1): 7-9. DOI: https://doi.org/10.12741/ebrasilis.v4i1.108 
Wagner, T. 2003. Seasonality of canopy beetles in Uganda. pp. 146-158. In: Basset, Y., V. Novotny, S. E. Miller \& R. L. Kitching (Eds.). Arthropods of tropical forests: spatio-temporal dynamics and resource use in the canopy. New York, Cambridge University Press.

Wickham, H. 2016. ggplot2: Elegant Graphics for Data Analysis. Springer-Verlag, New York. Version 4.0. Disponível em: < https:// ggplot2.tidyverse.org $>$. Acesso em 28 jun. 2020.

Editor Cientifico / Scientific Editor: Oscar Alexander Aguirre-Obando, UNIQUINDÍO, Colômbia

Recebido / Recibido / Received: 07.09.2020

Revisado / Revised: 11.12.2020

Aceito / Aceptado / Accepted: 03.02.2021

Publicado / Published: 17.02.2021

DOI: https://doi.org/10.5216/rbn.v18i1.65005

Dados disponíveis / Datos disponibles / Available data: Repositório não informado 